\title{
RELIABILITY ANALYSIS OF COMPLEX COMMUNICATION SYSTEMS*
}

\author{
J. Sztrik (Debrecen, Hungary)
}

UDC 519.2

This paper deals with a first-come, first-served(FCFS) queueing model to analyze the asymptotic behavior of a heterogeneous finite-source communication system with a single processor. Each source and the processor are assumed to operate in independent random environments, allowing the arrival and service processes to be Markov-modulated ones. Each message is characterized by its oun exponentially distributed source and processing time with parameter, depending on the state of the corresponding environment, that is, the arrival and service rates are subject to random fluctuations. Assuming that the arrival rates of the messages are many times greater than their service rates ("fast" arrival), it is shown that the time to the first system failure converges in distribution, under appropriate norming, to an exponentially distributed random variable. Some simple examples are considered to illustrate the effectiveness of the method proposed by comparing the approximate results to the exact ones.

\section{Introduction}

Performance evaluation of information-system development becomes more complex as the size and complexity of the system increases (c.f., $[7,11,17])$. Reliability is certainly one of the most important characteristics for communication networks. The measure of greatest interest is the distribution of the time to the first system failure. It is well known that the majority of problems can be treated with the help of semi-Markov processes (SMP). Since the failure-free operation time of the system corresponds to sojourn time problems, we can use the results obtained for SMP. If the exit from a given subset of the state space is a "rare" event, that is, it occurs with a small probability, it is natural to investigate the asymptotic behavior of the sojourn time in that subspace (see $[5,6,8,10]$ ). Realistic consideration of certain stochastic systems, however, often requires the introduction of a random environment, sometimes referred as to Markov-modulation, where system parameters are subjected to randomly occurring fluctuations. This situation may be attributed to certain changes in the physical environment such as weather, or sudden personal changes and work load alterations. In [4], Gaver proposed an efficient computational approach for the analysis of a generalized structure involving finite-state-space birth-and-death processes in a Markovian environment. Stern and Elwalid in [12] used Markov-modulated processes for analyzing some infinite-source information systems. This paper deals with a first-come, first-served (FCFS) queueing model to analyze the asymptotic behavior of a heterogeneous finite-source communication system with a single processor. The sources and the processor are assumed to operate in independent random environments. Each message is characterized by its own exponentially distributed source and processing time with parameter, depending on the state of the corresponding environment. Assuming that the arrival rates of the messages are many times greater than their service rates ("fast" arrival), it is shown that the time to the first system failure converges in distribution, under appropriate norming, to an exponentially distributed random variable. Some simple examples are considered to illustrate the effectiveness of the method proposed by comparing the approximate characteristics to the exact ones. This paper generalizes the results of Sztrik and Lukashuk [15] and Sztrik and Rigo [1C], where the sources are homogeneous and the whole system is governed by a single random environment and two random environments, respectively. The technique used here is similar to the one applied in [9] and [13,14].

\section{Preliminary Results}

In this section, a brief survey is given of the most related theoretical results, mainly due to Anisimov (see [1-3]), to be applied later on.

Let $\left(X_{\varepsilon}(k), k \geq 0\right)$ be a Markov chain with state space

$$
\bigcup_{q=0}^{m+1} X_{q}, \quad X_{i} \cap X_{j}=0, \quad i \neq j
$$

defined by the transition matrix $\left(p_{\varepsilon}\left(i^{(q)}, j^{(z)}\right)\right)$, satisfying the following conditions:

* Supported by the Hungarian National Foundation for Scientific Research (grant No. OTKA T14974/95).

Proceedings of the Seminar on Stability Problems for Stochastic Models, Vologda, Russia, 1998, Part II. 
1. $p_{\varepsilon}\left(i^{(0)}, j^{(0)}\right) \rightarrow p_{0}\left(i^{(0)}, j^{(0)}\right)$ as $\varepsilon \rightarrow 0, i^{(0)}, j^{(0)} \in X_{0}$, and matrix $P_{0}=\left(p_{0}\left(i^{(0)}, j^{(0)}\right)\right)$ is irreducible;

2. $p_{\varepsilon}\left(i^{(q)}, j^{(q+1)}\right)=\varepsilon \alpha^{(q)}\left(i^{(q)}, j^{(q+1)}\right)+o(\varepsilon), i^{(q)} \in X_{q}, j^{(q+1)} \in X_{q+1}$;

3. $p_{\varepsilon}\left(i^{(q)}, f^{(q)}\right) \rightarrow 0$ as $\varepsilon \rightarrow 0, i^{(q)}, f^{(q)} \in X_{q}, q \geq 1$

4. $p_{\varepsilon}\left(i^{(q)}, f^{(z)}\right) \equiv 0, i^{(q)} \in X_{q}, f^{(z)} \in X_{z}, z-f \geq 2$.

In the sequel, the set of states $X_{q}$ is called the $q$ th level of the chain, $q=1, \ldots, m+1$. Let us distinguish the subset of states

$$
\left\langle\alpha_{m}\right\rangle=\bigcup_{q=0}^{m} X_{q}
$$

Denote by $\left\{\pi_{\varepsilon}\left(i^{(q)}\right), i^{(q)} \in X_{q}\right\}, q=1, \ldots, m$, the stationary distribution of a chain with transition matrix

$$
\left(p_{\varepsilon}\left(i^{(q)}, j^{(z)}\right)\right) /\left(1-\sum_{k^{(m+1)} \in X_{m+1}} p_{\varepsilon}\left(i^{(q)}, k^{(m+1)}\right)\right), \quad i^{(q)} \in X_{q}, \quad j^{(z)} \in X_{z}, \quad q, z \leq m .
$$

Furthermore, denote by $g_{\varepsilon}\left(\left\langle\alpha_{m}\right)\right)$ the steady state probability of exit from $\left\langle\alpha_{m}\right\rangle$, that is,

$$
g_{\varepsilon}\left(\left\langle\alpha_{m}\right\rangle\right)=\sum_{i(m) \in X_{m}} \pi_{\varepsilon}\left(i^{(m)}\right) \sum_{j(m+1) \in X_{m+1}} p_{\varepsilon}\left(i^{(m)}, j^{(m+1)}\right)
$$

Denote by $\left\{\pi_{0}\left(i^{(0)}\right), i^{(0)} \in X_{0}\right\}$ the stationary distribution corresponding to $P_{0}$, and let

$$
\bar{\pi}_{0}=\left\{\pi_{0}\left(i^{(0)}\right), i^{(0)} \in X_{0}\right\}, \quad \bar{\pi}_{\varepsilon}^{(q)}=\left\{\pi_{E}\left(i^{(q)}\right), i^{(q)} \in X_{q}\right\}
$$

be row vectors. Finally, let the matrix

$$
A^{(q)}=\left(\dot{\alpha}^{(q)}\left(i^{(q)}, j^{(q+1)}\right)\right), \quad i^{(q)} \in X_{q}, \quad j^{(q+1)} \in X_{q+1}, \quad q=0, \ldots, m,
$$

be defined by Condition 2 .

Conditions 1-4 allow us to compute the main terms of the asymptotic expression for $\bar{\pi}_{\varepsilon}^{(q)}$ and $g_{\varepsilon}\left(\left\langle\alpha_{m}\right\rangle\right)$. Namely, we obtain

$$
\begin{gathered}
\bar{\pi}_{\varepsilon}^{(q)}=\varepsilon^{q} \bar{\pi}_{0} A^{(0)} A^{(1)} \ldots A^{(q-1)}+o\left(\varepsilon^{q}\right), \quad q=1, \ldots, m, \\
g_{\varepsilon}\left(\left\langle\alpha_{m}\right\rangle\right)=\varepsilon^{m+1} \bar{\pi}_{0} A^{(0)} A^{(1)} \ldots A^{(m)} \underline{1}+o\left(\varepsilon^{m+1}\right),
\end{gathered}
$$

where $\underline{1}=(1, \ldots, 1)^{*}$ is a column vector. Let $\left(\eta_{k}(t), t \geq 0\right)$ be an SMP given by the embedded Markov chain $\left(X_{\varepsilon}(k), k \geq 0\right)$, satisfying Conditions $1-4$. Let the times $\tau_{\varepsilon}\left(j^{(s)}, k^{(z)}\right)$ - transition times from state $j^{(s)}$ to state $k^{(z)}$ - satisfy the condition

$$
\mathrm{E} \exp \left\{i \theta \beta_{\varepsilon} \tau_{\varepsilon}\left(j^{(s)}, k^{(z)}\right)\right\}=1+a_{j k}(s, z, \theta) \varepsilon^{m+1}+o\left(\varepsilon^{m+1}\right), \quad i^{2}=-1,
$$

where $\beta_{\varepsilon}$ is some normalizing factor. Denote by $\Omega_{\varepsilon}(m)$ the instant at which the SMIP reaches the $(m+1)$ th level for the first time, the exit time from $\left\langle\alpha_{m}\right\rangle$, provided that $\eta_{\varepsilon}(0) \in\left\langle\alpha_{m}\right\rangle$. Then we have:

THEOREM 1 (cf. Anisimov [2]). If the above conditions are satisfied, then

$$
\lim _{\varepsilon \rightarrow 0} \mathbf{E} \exp \left\{i \theta \beta_{\epsilon} \Omega_{\varepsilon}(m)\right\}=(1-A(\theta))^{-1}
$$

where

$$
A(\theta)=\left(\sum_{j(0), k^{(0)} \in X_{0}} \pi_{0}\left(j^{(0)}\right) p_{0}\left(j^{(0)}, k^{(0)}\right) a_{j k}(0,0, \theta)\right) /\left(\bar{\pi}_{0} A^{(0)} A^{(\xi)} \ldots A^{(m)} \underline{1}\right)
$$

Corollary 1. In particular, if $a_{j k}(s, z, \theta)=i \theta m_{j k}(s, z)$, then the limit is an exponentially distributed random variable with mean

$$
\left(\sum_{j^{(0)}, k^{(0)} \in X_{0}} \pi_{0}\left(j^{(0)}\right) p_{0}\left(j^{(0)}, k^{(0)}\right) m_{j k}(0,0)\right) /\left(\bar{\pi}_{0} A^{(0)} A^{(1)} \ldots A^{(m)} \underline{1}\right)
$$




\section{The Queueing Model}

Consider a finite-source communication system with $N$ heterogeneous sources and a single processor. The sources and the receiver operate in independent random environments. The environmental changes are reflected in the values of the access and service rates that prevail at any point of time. The main objective is to adapt these parameters to respond to random changes effectively and thus maintain the derived level of system performance.

The source $p$ is assumed to operate in a random environment governed by an ergodic Markov chain $\left(\xi_{p}(t), t \geq 0\right)$ with state space $\left(1, \ldots, r_{p}\right)$ and with transition density matrix

$$
\left(a_{i_{p} j_{p}}^{(p)}, i_{p}, j_{p}=1, \ldots, r_{p}, a_{i_{p} j_{p}}^{(p)}=\sum_{k \neq i_{p}} a_{i_{p} k}^{(p)}\right)
$$

Whenever the environmental process $\xi_{p}(t)$ is in state $i_{p}$ the probability that the source generates a request in the time interval $(t, t+h)$ is $\lambda_{p}\left(i_{p}, \varepsilon\right)+o(h), p=1, \ldots, N$. Each message is transmitted to a receiver, where the service immediately starts if the processor is idle; otherwise, a queueing line is formed. The service discipline is first-come, first-served (FCFS). The receiver is also assumed to operate in a random environment governed by an ergodic Markov chain $(\xi(t), t \geq 0)$ with state space $\left(1, \ldots, r_{N+1}\right)$ and with transition density matrix

$$
\left(a_{i_{N+1} j_{N+1}}^{(N+1)}, i_{N+1}, j_{N+1}=1, \ldots, r_{N+1}, a_{i_{N+1} i_{N+1}}^{(N+1)}=\sum_{k \neq i_{N+1}} a_{i_{N+1} k}^{(N+1)}\right) .
$$

Whenever the environmental process $\xi_{N+1}(t)$ is in state $i_{p}$ the probability that the service of message $p$ is completed in the time interval $(t, t+h)$ is $\mu_{p}\left(i_{N+1}\right) h+o(h)$. If a given source has sent a message, it stays idle and it cannot generate another one. After being serviced each message immediately returns to its source, which hence becomes active. All random variables involved here and the random environments are assumed to be independent of each other.

In practical applications, it is very important to know the distribution of time until the receiver becomes empty, which is actually the busy period of the processor, for example, if it is a satellite, an aircraft, or any flying object that needs orientation.

Let us consider the system under the assumption of "fast" arrivals, i.e., $\lambda_{p}\left(i_{p}, \varepsilon\right) \rightarrow \infty$ as $\varepsilon \rightarrow 0$. For simplicity, let $\lambda_{p}\left(i_{p}, \varepsilon\right)=\lambda_{p}\left(i_{p}\right) / \varepsilon, p=1,1, \ldots, N$.

Denote by $Y_{\varepsilon}(t)$ the number of active sources at time $t$, and let

$$
\Omega_{\varepsilon}(m)=\inf \left\{t: t>0, Y_{\varepsilon}(t)=m+1 / Y_{\varepsilon}(0) \leq m\right\},
$$

that is, the instant at which the number of active sources reaches the $(m+1)$ th level for the first time, provided that at the beginning their number is no greater than $m, m=0, \ldots, N-1$. In the following, $\Omega_{\varepsilon}(m)$ is referred to as the time to the first system failure. In particular, for $m=N-1$ we get the case where the processor becomes idle, i.e., $\Omega_{\varepsilon}(N-1)$ is the busy period length of the receiver, or processor.

Denote by $\pi_{0}\left(i_{1}, i_{2}, \ldots, i_{N}, i_{N+1}: 0 ; k_{1}, \ldots, k_{N}\right)$ the steady-state probability that random environment $\xi_{p}(t)$ is in state $i_{p}, p=1, \ldots, N+1$, there is no active source, and the order of arrival of messages to the processor is $\left(k_{1}, \ldots, k_{N}\right)$. Similarly, denote by $\pi_{0}\left(i_{1}, i_{2}, \ldots, i_{N}, i_{N+1}: 1 ; k_{2}, \ldots, k_{N}\right)$ the steady-state probability that the $p$ th random environment is in state $i_{p}, p=1, \ldots, N+1$, source $k_{1}$ is active, and the other sources sent their messages in the order $\left(k_{2}, \ldots, k_{N}\right)$. Clearly, $\left(k_{s}, \ldots, k_{N}\right) \in V_{N}^{N-s+1} ; s=1,2$, where $V_{N}^{N-s+1}$ denotes the set of all variations of order $N-s+1$ of elements $1, \ldots, N$. Now we have:

THEOREM 2. For the system in question, under the above assumptions, independently of the initial state, the distribution of the normalized random variable $\varepsilon^{m} \Omega_{\varepsilon}(m)$ converges weakly to an exponentially distributed random variable with parameter

$$
\begin{gathered}
\Lambda=\sum_{i_{1}=1}^{r_{1}} \cdots \sum_{i_{N+1}=1}^{r_{N+1}} \sum_{\left(k_{1}, \ldots, k_{N}\right) \in V_{N}} \pi_{0}\left(i_{1}, \ldots, i_{N+1}: 1 ; k_{2}, \ldots, k_{N}\right) \\
\times \frac{\mu_{k_{3}}\left(i_{N+1}\right)}{\lambda_{k_{1}}\left(i_{k_{1}}\right)} \times \frac{\mu_{k_{3}}\left(i_{N+1}\right)}{\lambda_{k_{1}}\left(i_{k_{1}}\right)+\lambda_{k_{2}}\left(i_{k_{2}}\right)} \times \cdots \times \frac{\mu_{k_{m+1}}\left(i_{N+1}\right)}{\lambda_{k_{1}}\left(i_{k_{1}}\right)+\cdots+\lambda_{k_{m}}\left(i_{k_{m}}\right)} \frac{1}{D},
\end{gathered}
$$

where

$$
D=\sum_{p=1}^{N+1} \sum_{j_{p}=1}^{r_{p}} \sum_{\substack{i_{p}=1 \\ i_{p} \neq j_{p}}}^{r_{p}} \sum_{\left(k_{1}, \ldots, k_{n}\right) \in V_{N}^{N}} \pi_{0}\left(i_{1}, \ldots, i_{N+1}: 0 ; k_{1}, \ldots, k_{N}\right) a_{i_{i}, j_{p}}^{(p)} /\left(\sum_{q=1}^{N+1} a_{i_{q} i_{p}}^{(q)}+\mu_{k_{1}}\left(i_{N+1}\right)\right)^{2} .
$$


Proof. Let us introduce the following stochastic process:

$$
Z_{\varepsilon}(t)=\left(\xi_{1}(t), \ldots, \xi_{N+1}(t): Y_{\varepsilon}(t) ; \beta_{1}(t), \ldots, \beta_{Y_{\varepsilon}(t)}\right)
$$

where $\beta_{1}(t), \ldots, \beta_{Y_{c}(t)}$ denote the indices of the messages in order of their arrival at the processor. It is easy to see that $\left(Z_{\varepsilon}(t), t \geq 0\right)$ is a multi-dimensional Markov chain with rather complex state space

$$
E=\left(\left(i_{1}, \ldots, i_{N+1}: s ; k_{1}, \ldots, k_{N-s}\right), i_{p}=1, \ldots, r_{p}, p=1, \ldots, N+1,\left(k_{1}, \ldots, k_{N-s}\right) \in V_{N}^{N-s}, s=0, \ldots, N\right)
$$

where $k_{0}=\{0\}$ by definition. Furthermore, let

$$
\left\langle\alpha_{m}\right\rangle=\left(\left(i_{1}, \ldots, i_{N+1}: s ; k_{1}, \ldots, k_{N-s}\right), i_{p}=1, \ldots, r_{p}, p=1, \ldots, N+1,\left(k_{1}, \ldots, k_{N-s}\right) \in V_{N}^{N-s}, s=0, \ldots, m\right)
$$

be a subset of the states. Hence, our aim is to determine the distribution of the first exit time of $Z_{\varepsilon}(t)$ from $\left\langle\alpha_{m}\right\rangle$, provided that $Z_{\varepsilon}(0) \leq\left\langle\alpha_{m}\right\rangle$. It can easily be verified that the transition probabilities in any time interval $(t, t+h)$ are the following:

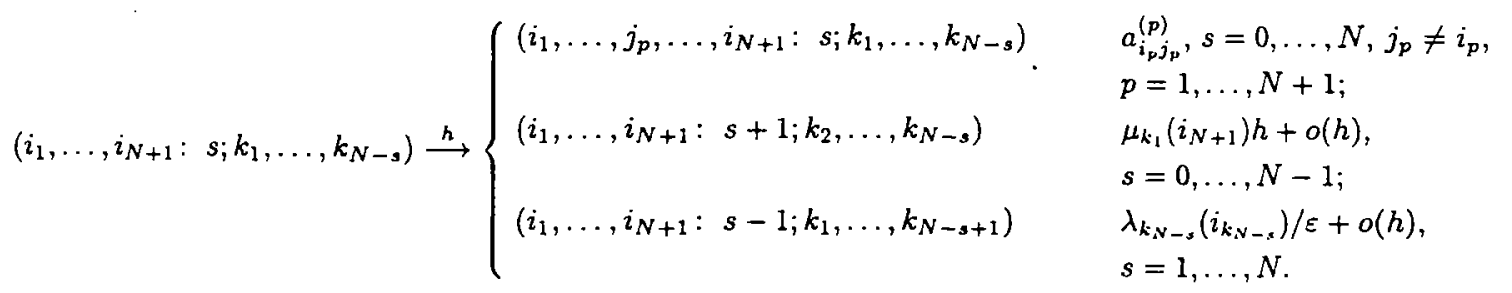

In addition, the sojourn time $\tau_{\varepsilon}\left(i_{1}, \ldots, i_{N+1}: s ; k_{1}, \ldots, k_{N-s}\right)$ of $Z_{\varepsilon}(t)$ in state $\left(i_{1}, \ldots, i_{N+1}: s ; k_{1}, \ldots, k_{N-s}\right)$ is exponentially distributed with parameter

$$
\gamma_{\varepsilon}\left(i_{1}, \ldots, i_{N+1}: s ; k_{1}, \ldots, k_{N-s}\right)=\left\{\begin{array}{lc}
a_{i_{1} i_{1}}^{(1)}+\cdots+a_{i_{N+1} i_{N+1}}^{(N+1)}+\mu_{k_{1}}\left(i_{N+1}\right), & s=0 \\
a_{i_{1} i_{1}}^{(1)}+\cdots+a_{i_{N+1} i_{N+1}}^{(N+1)}+\sum_{j \neq k_{1}, \ldots, k_{N-s}} \lambda_{j}\left(i_{j}\right) / \varepsilon+\mu_{k_{1}}\left(i_{N+1}\right), & s=1, \ldots, N-1 ; \\
a_{i_{1} i_{1}}^{(1)}+\cdots+a_{i_{N+1} i_{N+1}}^{(N+1)}+\sum_{j=1}^{N} \lambda_{j}\left(i_{j}\right) / \varepsilon, & s=N .
\end{array}\right.
$$

Thus, the transition probabilities for the embedded Markov chain are

$$
\begin{aligned}
& p_{\varepsilon}\left[\left(i_{1}, \ldots, i_{N+1}: s ; k_{1}, \ldots, k_{N-s}\right),\left(i_{1}, \ldots, j_{p}, \ldots, i_{N+1}: s ; k_{1}, \ldots, k_{N-s}\right)\right] \\
& =\frac{a_{i_{p}, j_{1}}^{(p)}}{\gamma_{\varepsilon}\left(i_{1}, \ldots, i_{N+1}: s ; k_{1}, \ldots, k_{N-s}\right)}, \quad s=0, \ldots, N, \quad p=1, \ldots, N+1 \\
& p_{\varepsilon}\left[\left(i_{1}, \ldots, i_{N+1}: s ; k_{2}, \ldots, k_{N-s}\right),\left(i_{1}, \ldots, i_{N+1}: s+1 ; k_{1}, \ldots, k_{N-s+1}\right)\right] \\
& =\frac{\mu_{k_{1}}\left(i_{N+1}\right)}{\gamma_{\varepsilon}\left(i_{1}, \ldots, i_{N+1}: s ; k_{1}, \ldots, k_{N-s}\right)}, \quad s=0, \ldots, N-1 ; \\
& p_{\varepsilon}\left[\left(i_{1}, \ldots, i_{N+1}: s ; k_{1}, \ldots, k_{N-s}\right),\left(i_{1}, \ldots, i_{N+1}: s-1 ; k_{1}, \ldots, k_{N-s-1}\right)\right] \\
& =\frac{\lambda_{k_{N-1}}\left(i_{k_{N-s}}\right) / \varepsilon}{\gamma_{\varepsilon}\left(i_{1}, \ldots, i_{N+1}: s ; k_{1}, \ldots, k_{N-s}\right)}, \quad s=1, \ldots, N .
\end{aligned}
$$

Thus, $\_\rightarrow 0$ implies

$$
\begin{gathered}
p_{\varepsilon}\left[\left(i_{1}, \ldots, i_{N+1}: 0 ; k_{1}, \ldots, k_{N}\right),\left(i_{1}, \ldots, j_{p}, \ldots, i_{N+1}: 0 ; k_{1}, \ldots, k_{N}\right)\right] \\
=\frac{a_{i_{p} j_{p}}^{(p)}}{a_{i_{1} i_{1}}^{(1)}+\cdots+a_{i_{N+1} i_{N+1}}^{(N+1)}+\mu_{k_{1}}\left(i_{N+1}\right)}, \quad p=1, \ldots, N+1
\end{gathered}
$$


$p_{\varepsilon}\left[\left(i_{1}, \ldots, i_{N+1}: s ; k_{1}, \ldots, k_{N-s}\right),\left(i_{1}, \ldots, j_{p}, \ldots, i_{N+1}: s ; k_{1}, \ldots, k_{N-s}\right)\right]=o(1), \quad s=1, \ldots, N, \quad p=1, \ldots, N+1$

$$
\begin{gathered}
p_{\varepsilon}\left[\left(i_{1}, \ldots, i_{N+1}: 0 ; k_{1}, \ldots, k_{N}\right),\left(i_{1}, \ldots, i_{N+1}: 1 ; k_{2}, \ldots, k_{N}\right)\right]=\frac{\mu_{k_{1}}\left(i_{N+1}\right)}{a_{i_{1} i_{1}}^{(1)}+\cdots+a_{i_{N+1} i_{N+1}}^{(N+1)}+\mu_{k_{1}}\left(i_{N+1}\right)} \\
p_{\varepsilon}\left[\left(i_{1}, \ldots, i_{N+1}: s ; k_{1}, \ldots, k_{N-s}\right),\left(i_{1}, \ldots, i_{N+1}: s+1 ; k_{2}, \ldots, k_{N-s}\right)\right] \\
=\frac{\mu_{k_{1}}\left(i_{N+1}\right) \varepsilon}{\sum_{p \neq k_{1}, \ldots, k_{N-s}} \lambda_{p}\left(i_{p}\right)}(1+o(1)), \quad s=1, \ldots, N-1 .
\end{gathered}
$$

This agrees with Conditions 1-4, but here the zero level is the set

$$
\left(\left(i_{1}, \ldots, i_{N+1}: s ; k_{1}, \ldots, k_{N-s}\right), i_{p}=1, \ldots, r_{p}, p=1, \ldots, N+1,\left(k_{1}, \ldots, k_{N-s}\right) \in V_{N}^{N-s}, s=0,1\right)
$$

and the $q$ th level is the set

$$
\left(\left(i_{1}, \ldots, i_{N+1}: q+1 ; k_{1}, \ldots, k_{N-q-1}\right), i_{p}=1, \ldots, r_{p}, p=1, \ldots, N+1,\left(k_{1}, \ldots, k_{N-q-1}\right) \in V_{N}^{N-q-1}\right) .
$$

Since the level 0 in the limit forms an essential class, the probabilities

$$
\begin{gathered}
\pi_{0}\left(i_{1}, \ldots, i_{N+1}: 0 ; k_{1}, \ldots, k_{N}\right), \quad \pi_{0}\left(i_{1}, \ldots, i_{N+1}: 1 ; k_{1}, \ldots, k_{N-1}\right), \\
i_{p}=1, \ldots, r_{p}, \quad p=1, \ldots, N+1, \quad\left(k_{1}, \ldots, k_{N-s}\right) \in V_{N}^{N-s}, \quad s=0,1,
\end{gathered}
$$

satisfy the system of equations

$$
\begin{gathered}
\pi_{0}\left(j_{1}, j_{2}, \ldots, j_{N+1}: 0 ; k_{1}, \ldots, k_{N}\right) \\
=\sum_{i_{1} \neq j_{1}} \pi_{0}\left(i_{1}, j_{2}, \ldots, j_{N+1}: 0 ; k_{1}, \ldots, k_{N}\right) a_{i_{1} j_{1}}^{(1)}\left(a_{i_{1} i_{1}}^{(1)}+a_{j_{2} j_{2}}^{(2)}+\cdots+a_{j_{N+1} j_{N+1}}^{(N+1)}+\mu_{k_{1}}\left(j_{N+1}\right)\right)^{-1} \\
+\sum_{i_{2} \neq j_{2}} \pi_{0}\left(j_{1}, j_{2}, \ldots, j_{N+1}: 0 ; k_{1}, \ldots, k_{N}\right) a_{i_{2} j_{2}}^{(2)}\left(a_{j_{1} j_{1}}^{(1)}+a_{i_{2} i_{2}}^{(2)}+\cdots+a_{j_{N+1} j_{N+1}}^{(N+1)}+\mu_{k_{1}}\left(j_{N+1}\right)\right)^{-1}+\ldots \\
+\sum_{i_{N+1} \neq j_{N+1}} \pi_{0}\left(j_{1}, j_{2}, \ldots, i_{N+1}: 0 ; k_{1}, \ldots, k_{N}\right) a_{i_{N+1} i_{N+1}}^{(N+1)}\left(a_{j_{1} j_{1}}^{(1)}+a_{j_{2} j_{2}}^{(2)}+\cdots+a_{i_{N+1} i_{N+1}}^{(N+1)}+\mu_{k_{1}}\left(i_{N+1}\right)\right)^{-1} \\
+\pi\left(j_{1}, \ldots, j_{N+1}: 1 ; k_{1}, \ldots, k_{N-1}\right), \\
\pi_{0}\left(j_{1}, \ldots, j_{N+1}: 1 ; k_{1}, \ldots, k_{N-1}\right)=\pi_{0}\left(j_{1}, \ldots, j_{N+1}: 0 ; k_{N}, k_{1}, \ldots, k_{N-1}\right) \\
\times \mu_{k_{N}}\left(j_{N+1}\right)\left(a_{j_{1} j_{1}}^{(1)}+\cdots+a_{j_{N+1} j_{N+1}}^{(N+1)}+\mu_{k_{N}}\left(j_{N+1}\right)\right)^{-1} .
\end{gathered}
$$

To apply (1) we need the solution of (2) and (3) with normalizing condition

$$
\sum_{p=1}^{N+1} \sum_{i_{p}=1}^{r_{p}} \sum_{\left(k_{1}, \ldots, k_{n}\right)}\left\{\pi_{0}\left(i_{1}, \ldots, i_{N+1}: 0 ; k_{1}, \ldots, k_{N}\right)+\pi_{0}\left(i_{1}, \ldots, i_{N+1}: 1 ; k_{1}, \ldots, k_{N-1}\right)\right\}=1 .
$$

Suppose that we have this solution. Then, by substituting it into (1), we get

$$
\begin{gathered}
g\left(\left\langle\alpha_{m}\right\rangle\right)=\varepsilon^{m} \sum_{i_{1}=1}^{r_{1}} \cdots \sum_{i_{N+1}=1}^{r_{N+1}} \sum_{\left(k_{1}, \ldots, k_{N}\right) \in V_{N}^{N}} \pi_{0}\left(i_{1}, \ldots, i_{N+1}: 1 ; k_{2}, \ldots, k_{N}\right) \\
\times \frac{\mu_{k_{2}}\left(i_{N+1}\right)}{\lambda_{k_{1}}\left(i_{k_{1}}\right)} \times \frac{\mu_{k_{3}}\left(i_{N+1}\right)}{\lambda_{k_{1}}\left(i_{k_{1}}\right)+\lambda_{k_{2}}\left(i_{k_{2}}\right)} \times \cdots \times \frac{\mu_{k_{m+1}}\left(i_{N+1}\right)}{\lambda_{k_{1}}\left(i_{k_{1}}\right)+\cdots+\lambda_{k_{m}}\left(i_{k_{m}}\right)}(1+o(1)) .
\end{gathered}
$$

Taking into account the exponentiality of $\tau_{\varepsilon}\left(i_{1}, \ldots, i_{N+1}: s ; k_{1}, \ldots, k_{N-s}\right)$ for fixed $\Theta$, we have

$$
\mathbf{E} \exp \left\{i \varepsilon^{m} \Theta \tau_{\varepsilon}\left(i_{1}, \ldots, i_{N+1}: 0 ; k_{1}, \ldots, k_{N-s}\right)\right\}=1+\varepsilon^{m} \frac{i \ominus}{a_{i_{1} i_{1}}^{(1)}+\cdots+a_{i_{N+1} i_{N+1}}^{(N+1)}+\mu_{k_{1}}\left(i_{N+1}\right)}(1+o(1))
$$


Observe that $\beta_{\varepsilon}=\varepsilon^{m}$ and, therefore, recalling Corollary 1 we immediately conclude that $\varepsilon^{m} \Omega_{\varepsilon}(m)$ converges weakly to an exponentially distributed random variable with parameter

$$
\begin{gathered}
\Lambda=\sum_{i_{1}=1}^{r_{1}} \cdots \sum_{i_{N+1}=1}^{r_{N+1}} \sum_{\left(k_{1}, \ldots, k_{n}\right) \in V_{N}^{N}} \pi_{0}\left(i_{1}, \ldots, i_{N+1}: 1 ; k_{2}, \ldots, k_{N}\right) \\
\times \frac{\mu_{k_{2}}\left(i_{N+1}\right)}{\lambda_{k_{1}}\left(i_{k_{1}}\right)} \times \frac{\mu_{k_{3}}\left(i_{N+1}\right)}{\lambda_{k_{1}}\left(i_{k_{1}}\right)+\lambda_{k_{2}}\left(i_{k_{2}}\right)} \times \cdots \times \frac{\mu_{k_{m+1}}\left(i_{N+1}\right)}{\lambda_{k_{1}}\left(i_{k_{1}}\right)+\cdots+\lambda_{k_{m}}\left(i_{k_{m}}\right)} \frac{1}{D},
\end{gathered}
$$

which completes the proof.

It should be noted that this asymptotic approach considerably reduces the calculations, since solving the system (2) and (3) is much easier than getting the stationary distribution of $\left(Z_{\varepsilon}(t), t \geq 0\right)$ and the system of stochastic relations concerning its sojourn time in $\left\langle\alpha_{m}\right\rangle$. Note that if $\mu_{p}\left(i_{N+1}\right)=\mu\left(i_{N+1}\right), p=1, \ldots, N$, a closed-form solution can be obtained. Namely, by substituting (3) into (2), we get

$$
\begin{gathered}
\pi_{0}\left(j_{1}, j_{2}, \ldots, j_{N+1}: 0 ; k_{1}, \ldots, k_{N}\right) \\
=\sum_{i_{1} \neq j_{1}} \pi_{0}\left(i_{1}, j_{2}, \ldots, j_{N+1}: 0 ; k_{1}, \ldots, k_{N}\right) a_{i_{1} j_{1}}^{(1)}\left(a_{i_{1} i_{1}}^{(1)}+a_{j_{2} j_{2}}^{(2)}+\cdots+a_{j_{N+1} j_{N+1}}^{(N+1)}+\mu\left(j_{N+1}\right)\right)^{-1} \\
+\sum_{i_{2} \neq j_{2}} \pi_{0}\left(j_{1}, j_{2}, \ldots, j_{N+1}: 0 ; k_{1}, \ldots, k_{N}\right) a_{i_{2} j_{2}}^{(2)}\left(a_{j_{1} j_{1}}^{(1)}+a_{i_{2} i_{2}}^{(2)}+\cdots+a_{j_{N+1} j_{N+1}}^{(N+1)}+\mu\left(j_{N+1}\right)\right)^{-1}+\ldots \\
+\sum_{i_{N+1} \neq j_{N+1}} \pi_{0}\left(j_{1}, j_{2}, \ldots, i_{N+1}: 0 ; k_{1}, \ldots, k_{N}\right) a_{i_{N+1} j_{N+1}}^{(N+1)}\left(a_{j_{1} j_{1}}^{(1)}+a_{j_{2} j_{2}}^{(2)}+\cdots+a_{i_{N+1} i_{N+1}}^{(N+1)}+\mu\left(i_{N+1}\right)\right)^{-1} \\
\quad+\pi_{0}\left(j_{1}, \ldots, j_{N+1}: 0 ; k_{N}, k_{1}, \ldots, k_{N-1}\right) \mu\left(j_{N+1}\right)\left(a_{j_{1} j_{1}}^{(1)}+\cdots+a_{j_{N+1} j_{N+1}}^{(N+1)}+\mu\left(j_{N+1}\right)\right)^{-1} .
\end{gathered}
$$

Denote by $\left(\pi_{i_{p}}^{(p)}, i_{p}=1, \ldots, r_{p}\right)$ the steady-state distribution of the governing Markov chains $\left(\xi_{p}(t), t \geq 0\right), p=$ $1, \ldots, N+1$, respectively. Clearly

$$
\pi_{j_{p}}^{(p)} a_{j_{\eta}, j_{p}}^{(p)}=\sum_{i_{p} \neq j_{p}} \pi_{i_{p}}^{(p)} a_{i_{p} j_{p}}^{(p)}, \quad p=1, \ldots, N+1
$$

It can be verified that the solution of (6) with (7) is

$$
\begin{gathered}
\pi_{0}\left(j_{1}, j_{2}, \ldots, j_{N+1}: 0 ; k_{1}, \ldots, k_{N}\right)=B\left(\pi_{j_{1}}^{(1)} \ldots \pi_{j_{N+1}}^{(N+1)}\right)\left(a_{j_{1} j_{1}}^{(1)}+\cdots+a_{j_{N+1} j_{N+1}}^{(N+1)}+\mu\left(j_{N+1}\right)\right), \\
\pi_{0}\left(j_{1}, \ldots, j_{N+1}: 1 ; k_{1}, \ldots, k_{N-1}\right)=B\left(\pi_{j_{1}}^{(1)} \ldots \pi_{j_{N+1}}^{(N+1)}\right) \mu\left(j_{N+1}\right)
\end{gathered}
$$

where $B$ is the normalizing constant, i.e.,

$$
\frac{1}{B}=N ! \sum_{i_{1}=1}^{r_{1}} \ldots \sum_{i_{N+1}=1}^{r_{N+1}}\left(\pi_{i_{1}}^{(1)} \ldots \pi_{i_{N+1}}^{(N+1)}\right)\left(a_{i_{1} i_{1}}^{(1)}+\cdots+a_{i_{N+1} i_{N+1}}^{(N+1)}+2 \mu\left(i_{N+1}\right)\right)
$$

Thus from (5) it follows that $\varepsilon^{m} \Omega(m)$ converges weakly to an exponentially distributed random variable with parameter

$$
\begin{gathered}
\Lambda=\frac{1}{N !} \sum_{i_{1}=1}^{r_{1}} \ldots \sum_{i_{N+1}=1}^{r_{N+1}} \sum_{\left(k_{1}, \ldots, k_{N}\right) \in V_{N}^{N}}\left(\pi_{i_{1}}^{(1)} \cdots \pi_{i_{N+1}}^{(N+1)}\right) \mu\left(i_{N+1}\right) \\
\times \frac{\mu\left(i_{N+1}\right)}{\lambda_{k_{1}}\left(i_{k_{1}}\right)} \times \frac{\mu\left(i_{N+1}\right)}{\lambda_{k_{1}}\left(i_{k_{1}}\right)+\lambda_{k_{2}}\left(i_{k_{2}}\right)} \times \cdots \times \frac{\mu\left(i_{N+1}\right)}{\lambda_{k_{1}}\left(i_{k_{1}}\right)+\cdots+\lambda_{k_{m}}\left(i_{k_{m}}\right)} .
\end{gathered}
$$

Consequently, the distribution of the time while the number of active sources reaches the $(m+1)$ th level for the first time is approximated by

$$
\mathbf{P}\left(\Omega_{\varepsilon}(m)>t\right)=\mathbf{P}\left(\varepsilon^{m} \Omega_{\varepsilon}(m)>\varepsilon^{m} t\right) \approx \exp \left(-\varepsilon^{m} \Lambda t\right) .
$$


In particular, when $m=N-1$ we get that the busy-period length of the receiver is asymptotically an exponentially distributed random variable with parameter

$$
\begin{aligned}
& \varepsilon^{N-1} \Lambda=\varepsilon^{N-1} \frac{1}{N !} \sum_{i_{1}=1}^{r_{1}} \cdots \sum_{i_{N+1}=1}^{r_{N+1}} \sum_{\left(k_{1}, \ldots, k_{N}\right) \in V_{N}^{N}}\left(\pi_{i_{1}}^{(1)} \ldots \pi_{i_{N+1}}^{(N+1)}\right) \mu\left(i_{N+1}\right) \\
& \times \frac{\mu\left(i_{N+1}\right)}{\lambda_{k_{1}}\left(i_{k_{1}}\right)} \times \frac{\mu\left(i_{N+1}\right)}{\lambda_{k_{1}}\left(i_{k_{1}}\right)+\lambda_{k_{2}}\left(i_{k_{2}}\right)} \times \cdots \times \frac{\mu\left(i_{N+1}\right)}{\lambda_{k_{1}}\left(i_{k_{1}}\right)+\cdots+\lambda_{k_{N-1}}\left(i_{k_{N-1}}\right)} .
\end{aligned}
$$

In the case where there are no random environments, that is, $\mu\left(i_{N+1}\right)=\mu, \lambda_{p}\left(i_{p}\right)=\lambda_{p}, i_{p}=1, \ldots, r_{p}, p=1, \ldots, N$, from (11) we get

$$
\varepsilon^{N-1} \Lambda=\varepsilon^{N-1} \frac{1}{N !} \sum_{\left(k_{1}, \ldots, k_{N}\right) \in V_{N}^{N}} \mu \frac{\mu}{\lambda_{k_{1}}} \frac{\mu}{\lambda_{k_{1}}+\lambda_{k_{2}}} \times \cdots \times \frac{\mu}{\lambda_{k_{1}}+\cdots+\lambda_{k_{N-1}}} .
$$

Finally, for the totally homogeneous case, from (12) we obtain

$$
\varepsilon^{N-1} \Lambda=\frac{1}{(N-1) !} \frac{\mu^{N}}{(\lambda / \varepsilon)^{N-1}} .
$$

The utilization $U_{r}$ of the receiver, which is the long-run fraction of time during which it is busy, can be given by

$$
U_{r}=\frac{1}{\varepsilon^{N-1} \Lambda}\left(\frac{1}{\varepsilon^{N-1} \Lambda}+I\right)^{-1}
$$

where $I$ denotes its idle-period length.

\section{Numerical Results}

This section presents a number of validation experiments (cf. Tables I-8) examining the credibility of the proposed approximation against exact results for the utilization at equilibrium. Note that, to the best of our knowledge, an exact formula for it is known only for homogeneous systems that are not affected by random environments, and it is given (via the Palm formula) by

$$
U_{r}^{*}=\sum_{k=1}^{N}\left(\begin{array}{l}
N \\
k
\end{array}\right) k ! \rho^{k} / \sum_{k=1}^{N}\left(\begin{array}{l}
N \\
k
\end{array}\right) k ! \rho^{k},
$$

where $\rho=(\lambda / \varepsilon) / \mu$. This is the reason why there are validation experiments for this case only. Instead of the overall utilization $U_{r}, U_{r}^{*}$, let us consider the utilization of the receiver with respect to the $p$ th source, denoted by $U_{p}$, $U_{p}^{*}$, respectively. Clearly $U_{p}=U_{r} / N$ and $U_{p}^{*}=U_{r}^{*} / N$, that is, they are so-called normalized utilizations. In this case, relations (13) and (14) reduce to the following approximation:

$$
U_{p}=\frac{1}{N} \frac{N !}{N !+(\mu /(\lambda / \varepsilon))^{N}}
$$

The numerical results can be seen in Tables 1-8. It can be observed that the approximate values for $\left\{U_{p}\right\}$ are very much comparable in accuracy to those provided by the exact results for $\left\{U_{p}^{*}\right\}$. However, the computational complexity, due to the proposed approximation, has been considerably reduced. As $\lambda / \varepsilon$ becomes greater than $\mu$, the $\left\{U_{p}\right\}$ approximations, as expected, approach the exact values of $\left\{U_{p}\right\}$. Clearly, the greater the number of sources the less the number of steps needed to reach the exact results.

TABLE $1 . N=3$.

\begin{tabular}{|c|c|c|}
\hline$\rho$ & $U_{P}^{*}$ & $U_{p}$ \\
\hline 1 & 0.3125 & 0.285714286 \\
\hline 2 & 0.329113924 & 0.326530612 \\
\hline $2^{3}$ & 0.332657201 & 0.332467532 \\
\hline $2^{3}$ & 0.333237575 & 0.333224862 \\
\hline $2^{4}$ & 0.333320592 & 0.333319771 \\
\hline $2^{5}$ & 0.333333169 & 0.333331638 \\
\hline $2^{6}$ & 0.333333125 & 0.333333121 \\
\hline $2^{4}$ & 0.333333307 & 0.333333307 \\
\hline $2^{8}$ & 0.333333333 & 0.333333333 \\
\hline
\end{tabular}


TABLE 2. $N=4$.

\begin{tabular}{|c|c|c|}
\hline$\rho$ & $U_{p}^{*}$ & $U_{p}$ \\
\hline 1 & 0.246153846 & 0.24 \\
\hline 2 & 0.249605055 & 0.249350649 \\
\hline $2^{2}$ & 0.249968310 & 0.249959317 \\
\hline $2^{3}$ & 0.249997756 & 0.249997457 \\
\hline $2^{4}$ & 0.249999999 & 0.249999999 \\
\hline $2^{5}$ & 0.25 & 0.25 \\
\hline
\end{tabular}

TABLE 3. $N=5$.

\begin{tabular}{|c|c|c|}
\hline$\rho$ & $U_{p}^{*}$ & $U_{p}$ \\
\hline 1 & 0.199386503 & 0.198347107 \\
\hline 2 & 0.199968409 & 0.199947930 \\
\hline $2^{2}$ & 0.199998732 & 0.199998372 \\
\hline $2^{3}$ & 0.199999955 & 0.199999949 \\
\hline $2^{4}$ & 0.199999998 & 0.199999998 \\
\hline $2^{5}$ & 0.2 & 0.2 \\
\hline
\end{tabular}

TABLE 4. $N=6$.

\begin{tabular}{|c|c|c|}
\hline$\rho$ & $U_{p}^{*}$ & $U_{p}$ \\
\hline 1 & 0.166581502 & 0.166435506 \\
\hline 2 & 0.166064473 & 0.166666305 \\
\hline $2^{2}$ & 0.166666623 & 0.166666661 \\
\hline $2^{3}$ & 0.166060660 & 0.166660666 \\
\hline
\end{tabular}

TABLE 5. $N=7$.

\begin{tabular}{|c|c|c|}
\hline$\rho$ & $U_{p}^{*}$ & $U_{p}$ \\
\hline 1 & 0.142846715 & 0.142828804 \\
\hline 2 & 0.142857009 & 0.142856921 \\
\hline $2^{2}$ & 0.142857142 & 0.142857141 \\
\hline $2^{3}$ & 0.142857143 & 0.142857143 \\
\hline
\end{tabular}

TABLE $6 . N=8$.

\begin{tabular}{|c|c|c|}
\hline$\rho$ & $U_{p}^{*}$ & $U_{p}$ \\
\hline 1 & 0.124998860 & 0.1249969 \\
\hline 2 & 0.124999993 & 0.124999988 \\
\hline $2^{2}$ & 0.125 & 0.125 \\
\hline
\end{tabular}

TABLE 7. $N=9$.

\begin{tabular}{|c|c|c|}
\hline$\rho$ & $U_{p}^{*}$ & $U_{p}$ \\
\hline 1 & 0.111110998 & 0.111110805 \\
\hline 2 & 0.111111111 & 0.111111111 \\
\hline
\end{tabular}

TABLE 8. $N=10$.

\begin{tabular}{|c|c|c|}
\hline$\rho$ & $U_{p}^{*}$ & $U_{p}$ \\
\hline 1 & 0.099999999 & 0.099999999 \\
\hline 2 & 0.1 & 0.1 \\
\hline
\end{tabular}

\section{Concluding Remarks}

In this paper, we presented a queueing model to analyze the behavior of an FCFS heterogeneous finite-source communication system with a single processor. The system operates in Markovian environments and the messages arrive fast compared to their service. An asymptotic approach was provided to obtain the distribution of the time to the first system failure. The credibility of the method is illustrated with some simple validation experiments, and favorable comparisons against exact results are made. 


\section{REFERENCES}

1. V. V. Anisimov, "Switching processes: Averaging principle, diffusion approximation, and applications," Act. Appl. Math., 40, 95-141 (1995).

2. V. V. Anisimov, "Asymptotic analysis of switching queueing systems under conditions of low and heavy loading; matrix-analytic methods in stochastic models," in: Lecture Notes in Pure and Application Mathematical Series, 183, Marcel Dekker (1996).

3. V. V. Anisimov and J. Sztrik, "Asymptotic analysis of some controlled finite-source queueing systems," Act. Cybern., 9, 27-38 (1989).

4. D. P. Gaver, P. A. Jacobs, and G. Latouche, "Finite birth-and-death models in randomly changing environments," Adv. Appl. Probab., 16, 715-731 (1984).

5. I. B. Gertsbakh, "Asymptotic methods in reliability theory: A review," Adv. Appl. Probab., 16, 147-175 (1984).

6. I. B. Gertsbakh, Statistical Reliability Theory, Marcel Dekker, New York (1989).

7. P. Harrison and N. M. Patel, Performance Modeling of Communication Networks and Computer Architectures, Addison-Wesley (1993).

8. J. Keilson, Markov Chain Models - Rarity and Exponentiality, Springer, Berlin (1979).

9. D. D. Kouvatsos, R. Fretwell, and J. Sztrik, "Bounds on the effects of correlation in a stable MMPP/MMPP/1/N queue: An asymptotic approach," in: Proceedings of Second Workshop on Performance Modeling and Evaluation of ATM Networks, Chapman and Hall, London (1995), pp. 261-281.

10. I. N. Kovalenko, "Rare events in queueing systems; A survey," Queueing Syst., 16, I-49 (1994).

11. I. Mitrani, Modeling of Computer and Communication Systems, Cambridge Univ. Press, Cambridge (1987).

12. T. E. Stern and A. I. Elwalid, "Analysis of separable Markov-modulated rate models for information-handling systems," Adv. Appl. Probab., 23, 105-139 (1989).

13. J. Sztrik, "Modeling of a multiprocessor system in a randomly changing environment," Perform. Evaluat., 17, 1-11 (1993).

14. J. Sztrik and D. D. Kouvatsos, "Asymptotic analysis of a heterogeneous multiprocessor system in a randomly changing environment," IEEE Trans. Soft. Engineer., 17, 1069-1075 (1991).

15. J. Sztrik and L. Lukashuk, "Modeling of a communication system evolving in a random environment," Acta Cybern., 10, 85-91 (1991).

16. J. Sztrik and R. Rigo, "On a closed communication system with fast sources and operating in Markovian environments," J. Inform. Process. Cybernet, EIK, 29, 241-240 (1993).

17. H. Takagi, "Bibliography on performance evaluation," in: Stochastic Analysis of Computer and Communication Systems, North-Holland, Amsterdam (1990).

Department of Mathematics, University of Debrecen,

P.O. Bot 12, 4010 Debrecen, Hungary

e-mail: jsztrik@math.klte.hu 